\title{
On finite volume effects in the chiral extrapolation of baryon masses
}

\author{
M.F.M. Lutz ${ }^{1}$, K. Schwarz ${ }^{1}$ and R. Bavontaweepanya ${ }^{2}$, C. Kobdaj ${ }^{2}$ \\ ${ }^{1}$ GSI, Darmstadt, Germany; ${ }^{2}$ Suranaree University of Technology, Nakhon Ratchasima, Thailand
}

We report on a comprehensive analysis of the available three flavour QCD lattice simulations of six different groups on the baryon octet and decuplet masses [1]. We obtained an accurate 12 parameter description of altogether more than 220 lattice data points, where we kept all data with pion masses smaller than $600 \mathrm{MeV}$. Our study extends previous works $[4,5,6]$ and is based on the relativistic three-flavour chiral Lagrangian with baryon octet and decuplet degrees of freedom. The baryon self energies were computed in a finite box at $\mathrm{N}^{3} \mathrm{LO}$, where the physical masses are kept inside all loop integrals [1,2]. The low-energy parameters were constrained by using large- $N_{c}$ sum rules [3].

Accurate predictions for all relevant low-energy parameters were obtained. In particular we extracted a pionnucleon sigma term of $(39 \pm 1) \mathrm{MeV}$ and a strangeness sigma term of the nucleon of $\sigma_{s N} \simeq(4 \pm 1) \mathrm{MeV}$. The flavour SU(3) chiral limit of the baryon octet and decuplet masses was determined with $(802 \pm 4) \mathrm{MeV}$ and $(1103 \pm 6)$ $\mathrm{MeV}$. In our fits we used the empirical masses of the baryon octet and decuplet states as a constraint. That allowed us to perform independent scale settings for the various lattice data. We obtained results for the lattice scales that are compatible with previous estimates, but appear to be much more accurate. Detailed predictions for the baryon masses as currently evaluated by the ETM lattice QCD group are made.
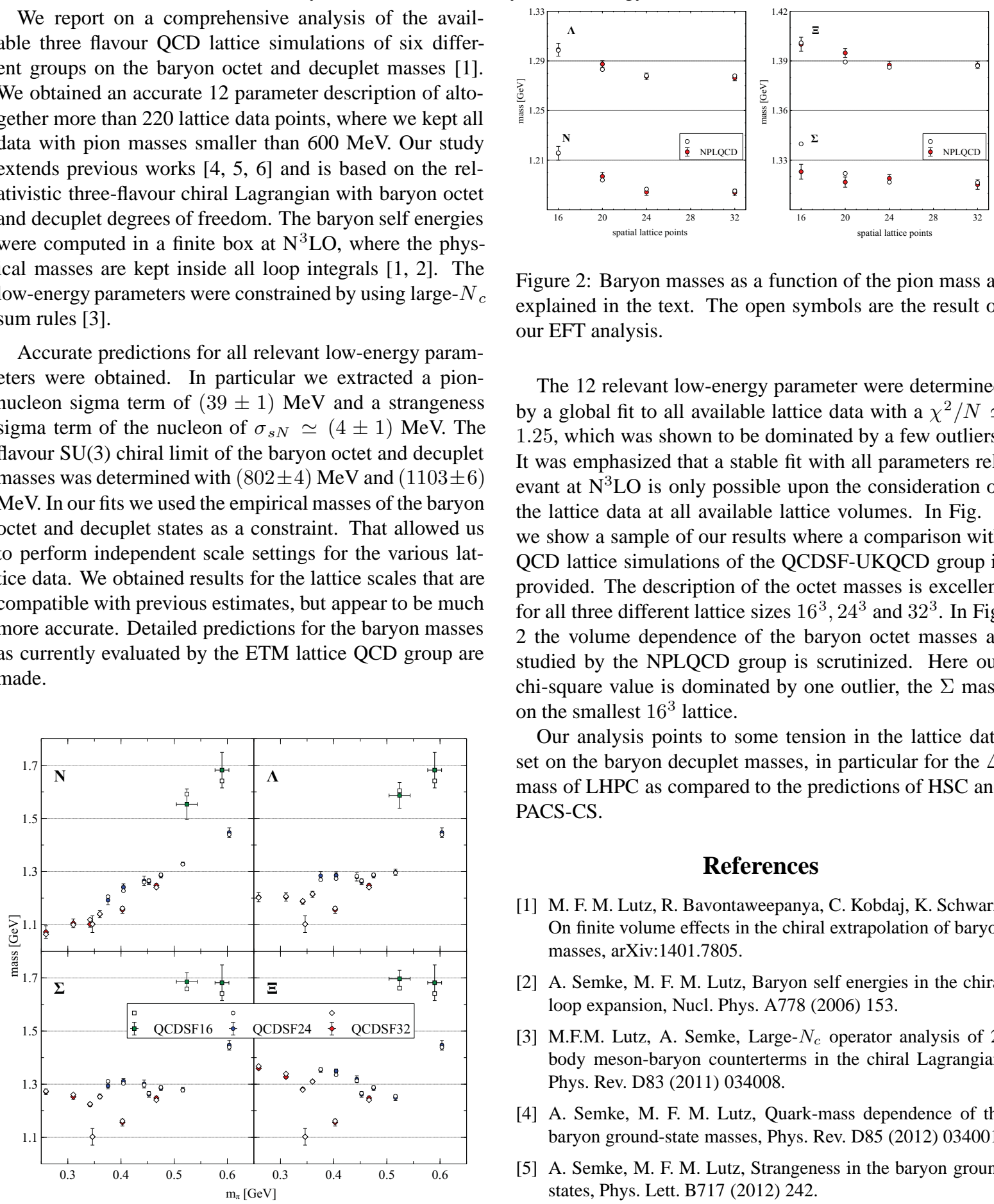

Figure 2: Baryon masses as a function of the pion mass as explained in the text. The open symbols are the result of our EFT analysis.

The 12 relevant low-energy parameter were determined by a global fit to all available lattice data with a $\chi^{2} / N \simeq$ 1.25 , which was shown to be dominated by a few outliers. It was emphasized that a stable fit with all parameters relevant at $\mathrm{N}^{3} \mathrm{LO}$ is only possible upon the consideration of the lattice data at all available lattice volumes. In Fig. 1 we show a sample of our results where a comparison with QCD lattice simulations of the QCDSF-UKQCD group is provided. The description of the octet masses is excellent for all three different lattice sizes $16^{3}, 24^{3}$ and $32^{3}$. In Fig. 2 the volume dependence of the baryon octet masses as studied by the NPLQCD group is scrutinized. Here our chi-square value is dominated by one outlier, the $\Sigma$ mass on the smallest $16^{3}$ lattice.

Our analysis points to some tension in the lattice data set on the baryon decuplet masses, in particular for the $\Delta$ mass of LHPC as compared to the predictions of HSC and PACS-CS.

\section{References}

[1] M. F. M. Lutz, R. Bavontaweepanya, C. Kobdaj, K. Schwarz, On finite volume effects in the chiral extrapolation of baryon masses, arXiv:1401.7805.

[2] A. Semke, M. F. M. Lutz, Baryon self energies in the chiral loop expansion, Nucl. Phys. A778 (2006) 153.

[3] M.F.M. Lutz, A. Semke, Large- $N_{c}$ operator analysis of 2body meson-baryon counterterms in the chiral Lagrangian, Phys. Rev. D83 (2011) 034008.

[4] A. Semke, M. F. M. Lutz, Quark-mass dependence of the baryon ground-state masses, Phys. Rev. D85 (2012) 034001.

[5] A. Semke, M. F. M. Lutz, Strangeness in the baryon ground states, Phys. Lett. B717 (2012) 242.

Figure 1: Baryon masses as a function of the pion mass as explained in the text. The open symbols are the result of our EFT analysis.

[6] M. F. M. Lutz, A. Semke, On the consistency of recent QCD lattice data of the baryon ground-state masses, Phys. Rev. D86 (2012) 091502. 